\title{
Astronomical optical frequency comb generation and test in a fiber-fed MUSE spectrograph
}

J. M. Chavez Boggio, T. Fremberg, B. Moralejo, M. Rutowska, E. Hernandez, et al.

J. M. Chavez Boggio, T. Fremberg, B. Moralejo, M. Rutowska, E. Hernandez, M. Zajnulina, A. Kelz, D. Bodenmüller, C. Sandin, M. Wysmolek, H. Sayinc, J. Neumann, R. Haynes, M. M. Roth, "Astronomical optical frequency comb generation and test in a fiber-fed MUSE spectrograph," Proc. SPIE 9151, Advances in Optical and Mechanical Technologies for Telescopes and Instrumentation, 915120 (28 July 2014); doi: 10.1117/12.2056684

EDent: SPIE Astronomical Telescopes + Instrumentation, 2014, Montréal, Quebec, Canada 


\title{
Astronomical optical frequency comb generation and test in a fiber-fed MUSE spectrograph
}

\author{
J.M. Chavez Boggio ${ }^{a}$, T. Fremberga, B. Moralejo ${ }^{a}$, M. Rutowska ${ }^{a}$, E. Hernandez ${ }^{\text {a }}$ M. Zajnulina ${ }^{a}$, A. \\ Kelz $^{\mathrm{a}}$, D. Bodenmüller ${ }^{\mathrm{a}}$, C. Sandin ${ }^{\mathrm{a}}$, M. Wysmolek ${ }^{\mathrm{c}}$, H. Sayinc ${ }^{\mathrm{b}}$, J. Neumann ${ }^{\mathrm{bc}}$, R. Haynes ${ }^{\mathrm{a}}$, and \\ M.M. Roth ${ }^{\mathrm{a}}$ \\ ainnoFSPEC-VKS, Leibniz-Institut für Astrophysik, An der Sternwarte 16, D-14482 Potsdam, \\ Germany \\ ${ }^{b}$ Laser Zentrum Hannover e.V., Hollerithallee 8, D-30419 Hannover, Germany \\ ${ }^{c}$ Centre for Quantum Engineering and Space-Time Research- QUEST, Welfengarten 1, \\ D-30167 Hannover, Germany
}

\begin{abstract}
We here report on recent progress on astronomical optical frequency comb generation at innoFSPEC-Potsdam and present preliminary test results using the fiber-fed Multi Unit Spectroscopic Explorer (MUSE) spectrograph. The frequency comb is generated by propagating two free-running lasers at 1554.3 and $1558.9 \mathrm{~nm}$ through two dispersionoptimized nonlinear fibers. The generated comb is centered at $1590 \mathrm{~nm}$ and comprises more than one hundred lines with an optical-signal-to-noise ratio larger than $30 \mathrm{~dB}$. A nonlinear crystal is used to frequency double the whole comb spectrum, which is efficiently converted into the $800 \mathrm{~nm}$ spectral band. We evaluate first the wavelength stability using an optical spectrum analyzer with $0.02 \mathrm{~nm}$ resolution and wavelength grid of $0.01 \mathrm{~nm}$. After confirming the stability within $0.01 \mathrm{~nm}$, we compare the spectra of the astro-comb and the Ne and $\mathrm{Hg}$ calibration lamps: the astro-comb exhibits a much larger number of lines than lamp calibration sources. A series of preliminary tests using a fiber-fed MUSE spectrograph are subsequently carried out with the main goal of assessing the equidistancy of the comb lines. Using a P3d data reduction software we determine the centroid and the width of each comb line (for each of the 400 fibers feeding the spectrograph): equidistancy is confirmed with an absolute accuracy of $0.4 \mathrm{pm}$.
\end{abstract}

Keywords: Astronomical wavelength calibration, spectrograph, optical frequency comb, dispersion engineering.

\section{INTRODUCTION}

The quest for improved wavelength calibration procedures for optical or near infrared (NIR) spectrographs at groundbased or space telescopes has received great attention over the last years [1-3]. A large amount of research has been focused on the calibration of high resolution spectrographs [4] because of their potentials for ground-breaking discoveries, e.g. a possible change of the fine structure constant over cosmic times [5], the direct measurement of the Hubble constant [6], or the detection of earth-like extra-solar planets [7]. To meet the high accuracy demands for such applications, the Astronomy and Photonic communities are joining efforts to develop astronomical laser frequency combs as an alternative calibration source to conventional lamps. This is currently done by using a fully stabilized modelocked laser followed by a set of two or three stabilized Fabry-Perot cavities to filter out unwanted comb modes and then a set of nonlinear stages for subsequent spectral broadening. While mode locked lasers offer equally spaced spectral lines with a few picometer spacing, astronomical spectrographs require a spacing of tens of pm (high resolution) or even a few nm (low resolution) [7-12]. The approach involving optical filtering to generate astronomical frequency combs (astro-combs) for high resolution applications has been pursued by a number of institutions worldwide and a number of on-sky tests have been carried out with success. For example, an astro-comb as a wavelength calibration source was tested at the High Accuracy Radial velocity Planet Searcher (HARPS) spectrograph with better radial velocity accuracies than with the conventional Ar lamp [10]. Using the same filtering approach an astro-comb centered at $1.5 \mu \mathrm{m}$ was recently demonstrated at NIST and calibration tests at the Pathfinder Spectrograph were performed [12]. While the onsky tests were performed only with high-resolution spectrographs, medium and low resolution spectrographs can also benefit from an improved calibration source. In particular for stellar population and the chemical evolution of stars applications. However, adapting the filtering approach to low and medium resolution applications is unrealistic because 
it will require filtering thousands of lines while maintaining a good optical-signal-to-noise ratio between unfiltered and filtered modes. Furthermorer, in order to massively deploy OFCs in telescopes around the world, the degree of complexity, size, and cost of actual astro-combs needs to be dramatically reduced.

Recently it has been proposed the use of the transmission function of a Fabry-Perot etalon to generate an array of lines [13]. This is done in conjunction with a white light source with bandwidth matching the spectrograph bandwidth. Even though this technique has been demonstrated by several groups and the resulting calibration source is expected to be deployed in a number of spectrographs, the exact location of each line depends on the Fabry Perot dispersion. Another approach for comb generation with large mode spacing consist in using high-Q micro-resonator [14]. In this paper we report on the ongoing research at innoFSPEC-Potsdam addressing calibration technologies for medium and low resolution astronomical spectrographs. In section II we describe the approach we follow for comb generation. Section 3 describes the preliminary results using a fiber-fed MUSE spetrograph. Finally, in section 4 we describe the conclusions.

\section{ASTRO-COMB GENERATION IN FIBERS}

Figure 1 shows the schematic of the nonlinear system to generate the astronomical optical frequency comb $[15,16]$. It consists of two equally intense lasers (with wavelengths $\lambda_{1}=1554.3 \mathrm{~nm}$ and $\lambda_{2}=1558.9 \mathrm{~nm}$ ) that are propagated through two nonlinear stages. The two-tone initial field corresponds temporally to a sinusoidal square pulse that is propagated first through an amplifying fibre that amplifies the average power and also provides strong (adiabatic) pulse compression. The aim is to generate clean sub-100 fs pulses with peak power larger than $1 \mathrm{~kW}$ for subsequent generation of astronomical optical frequency combs in a low-dispersion nonlinear fibre. A cascade of four-wave-mixing (FWM) products is generated after propagating through a $1.5 \mathrm{~m}$ long fiber having low dispersion $\left(\beta_{2}=0.05 \mathrm{ps}^{2} / \mathrm{km}, \beta_{3}=0.0081\right.$ $\left.\mathrm{ps}^{3} / \mathrm{km}\right)$ and high nonlinearity $\left(\gamma=10(\mathrm{~W}-\mathrm{km})^{-1}\right)$.

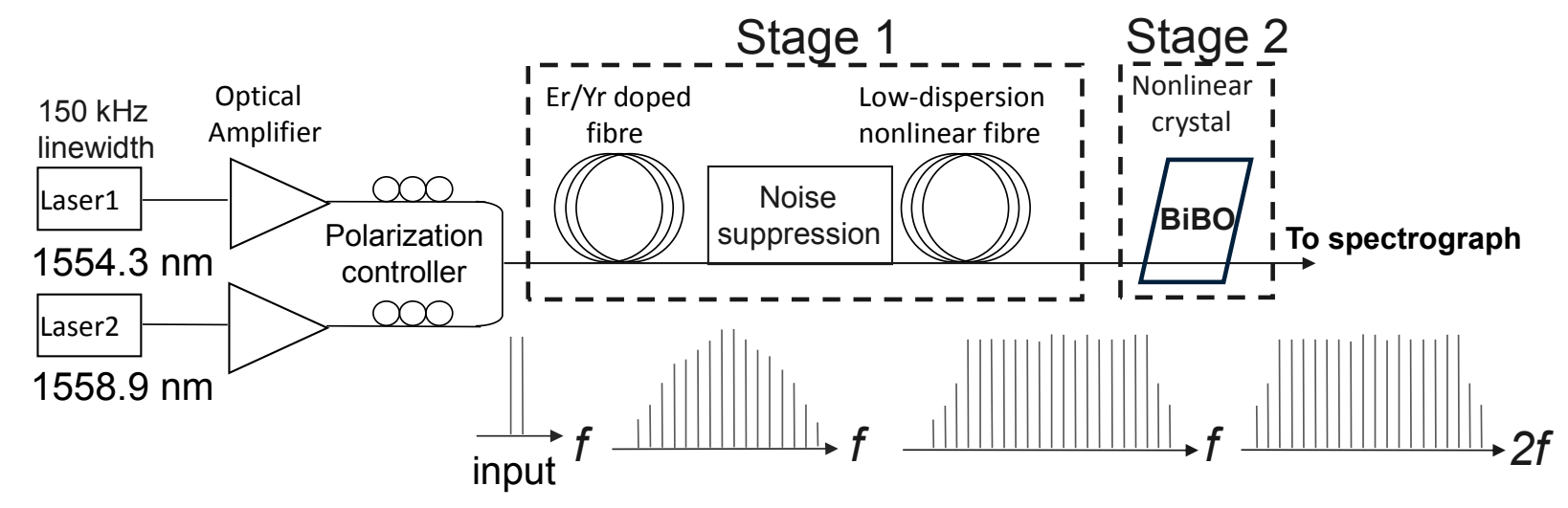

Figure 1. Schematic of the proposed astronomical frequency generator.

The optical frequency comb generated through the cascade of FWM products is centered at $1600 \mathrm{~nm}$ and extends over a bandwidth of $60 \mathrm{THz}$. The optical signal to noise ratio (OSNR) is larger than $30 \mathrm{~dB}$ for nearly the entire comb bandwidth. Figure 2 (left) shows a zoom of the spectrum of the generated astronomical comb where a good intensity equalization can be noted. The comb was subsequently propagated through a BiBO nonlinear crystal for frequency doubling. The replicated comb is now centered at $800 \mathrm{~nm}$ and a zoom of its spectrum is shown in Figure 2 (right). Even though the power carried by each comb line is not equalized along the whole comb bandwidth (power variation is 20 $\mathrm{dB}$ ), the noise characteristics and bandwidth are adequate to carry out tests with the fiber-fed MUSE spectrograph. 

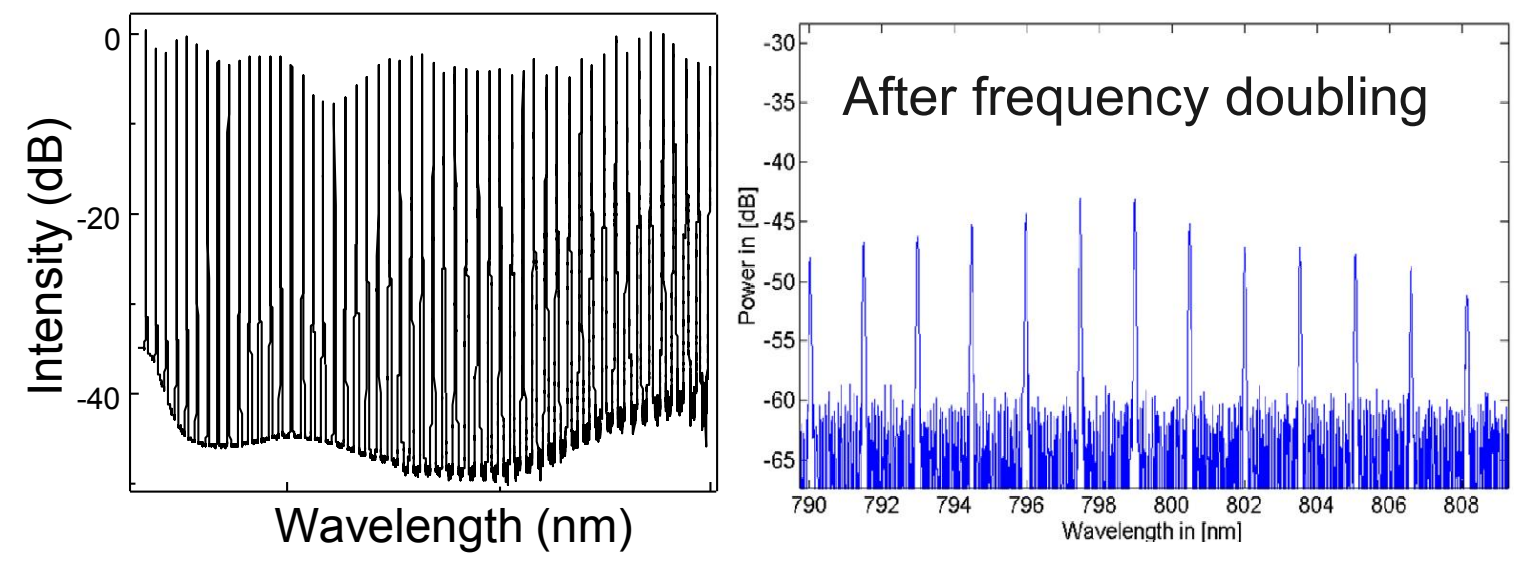

Figure 2. Left: Zoom of output spectrum around $1600 \mathrm{~nm}$. Right: Zoom of output spectrum around $800 \mathrm{~nm}$.

The first test was checking the wavelength stability of each line of the frequency comb centered at 1600 and at $800 \mathrm{~nm}$. We use an optical spectrum analyzer (OSA) having $0.02 \mathrm{~nm}$ resolution and wavelength grid of $0.01 \mathrm{~nm}$. Figure 3 shows the measured central wavelength for a time frame of 270 seconds (tests involving a few hours time-frame were also performed). It was observed that the maximum change of the central wavelength was $0.01 \mathrm{~nm}$. This measured change of the central wavelength, since it is within the resolution of the OSA, is the upper limit of the actual wavelength fluctuation.
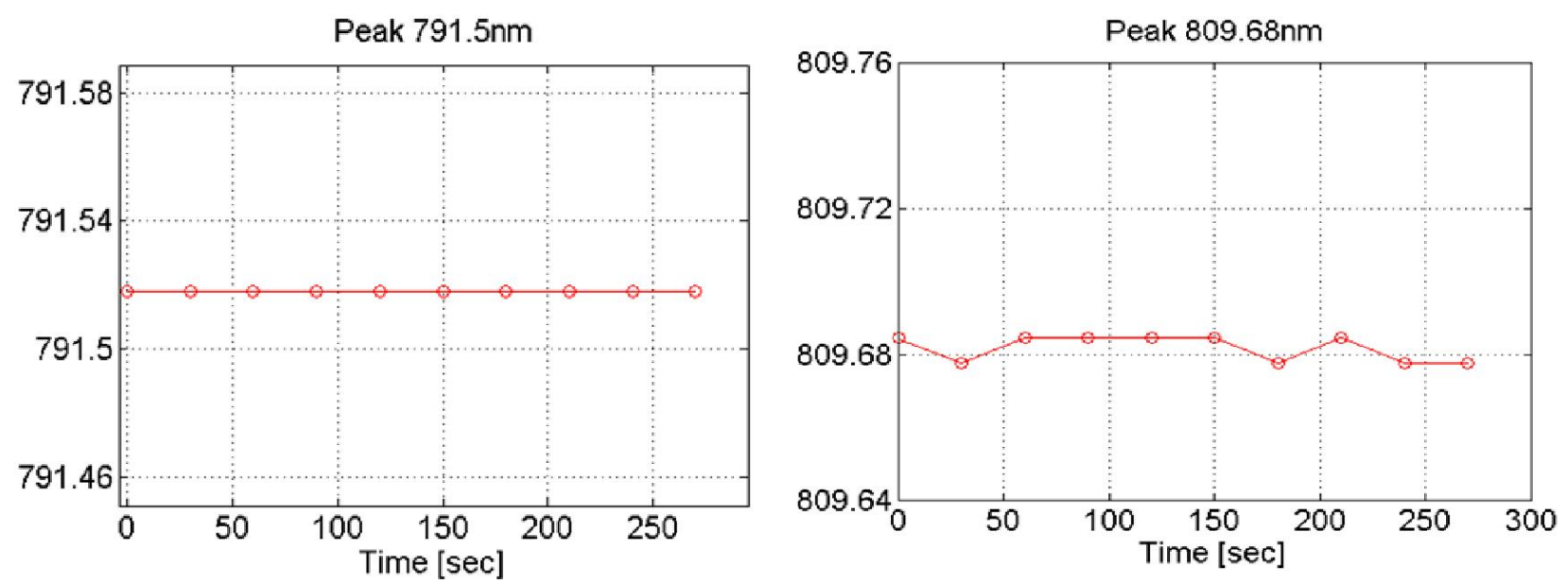

Figure 3. Central wavelength as a function of time for a comb wavelength at $791.5 \mathrm{~nm}$ (Left) and $809.68 \mathrm{~nm}$ (Right).

\section{PRELIMINARY TEST USING A MUSE SPECTROGRAPH}

Preliminary tests with the generated frequency comb are carried out using a fiber-fed MUSE spectrograph. The spectrograph, shown in Fig. 4, seeks to combine a broadband optical spectrograph with a new generation of multi-object deployable fiber bundles. The modification of the spectrograph of the Multi-Unit Spectroscopic Explorer, which is a VLT integral-field instrument using slicers, is performed by using a $20 \times 20$ fiber-fed input. The MUSE spectrograph operates at a wavelength range from $465 \mathrm{~nm}$ to $930 \mathrm{~nm}$ using a $4096 \mathrm{x} 409615 \mu \mathrm{m}$ pixel CCD detector. The wavelength calibration is routinely done with $\mathrm{Ne}$ and $\mathrm{Hg}$ lamps. 

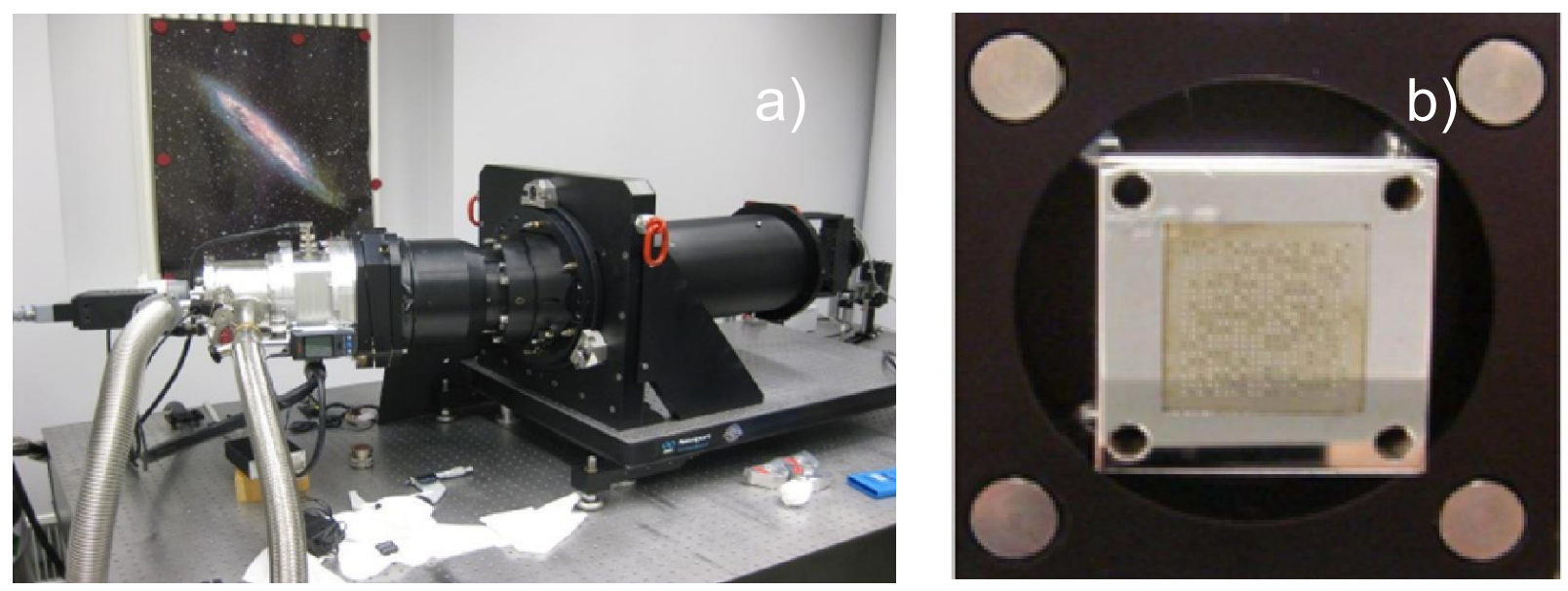

Figure 4. (a) Fiber-fed MUSE spectrograph. (b) $20 \times 20$ fiber input.

Astro-comb spectra were taken at regular time intervals and with different intensities in order to not saturate the CCD detector. A comparison between the spectrum of the Ne lamp and the astro-comb is shown in Figure 5 for two different spectral regions: the advantage of the astro-comb is that it provides a much larger number of calibration lines.

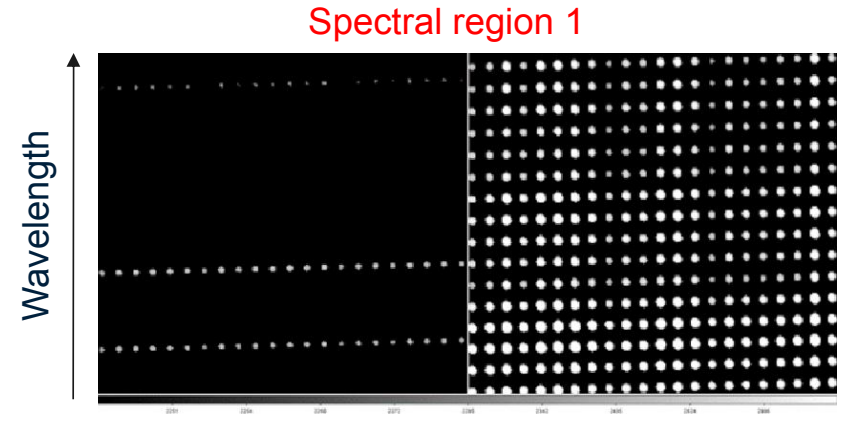

Ne lamp

Astro-comb

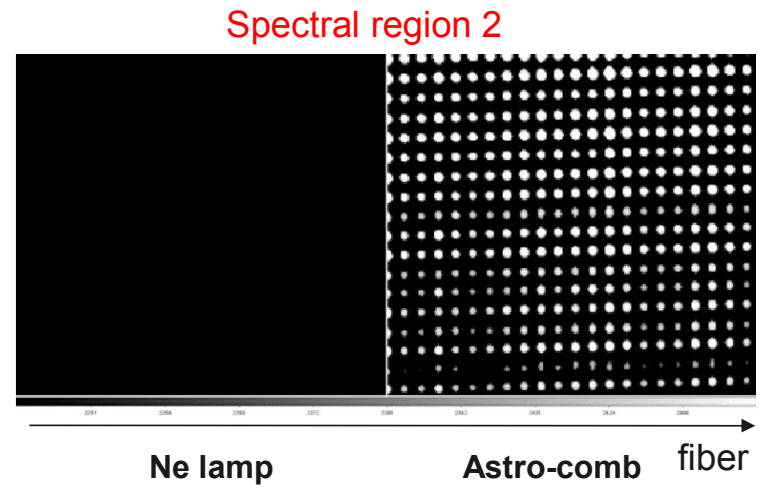

Figure 5. Comparison of the spectra of $\mathrm{Ne}$ and astro-comb light for two different regions. Vertical axis is wavelength while the horizontal one is fiber.

In the present version of generated astro-comb spectrum it comprised around 100 lines. Each one was independently fitted using a Gaussian function. Figure 6 shows the zoom of one spectrum illustrating the fitting of two adjacent comb lines. Note that each comb line is sampled/detected by 5 pixels. The fitting allows us to determine the centroid and the width of each line. This was performed for all the comb lines and for a representative number of the 400 fibers. 


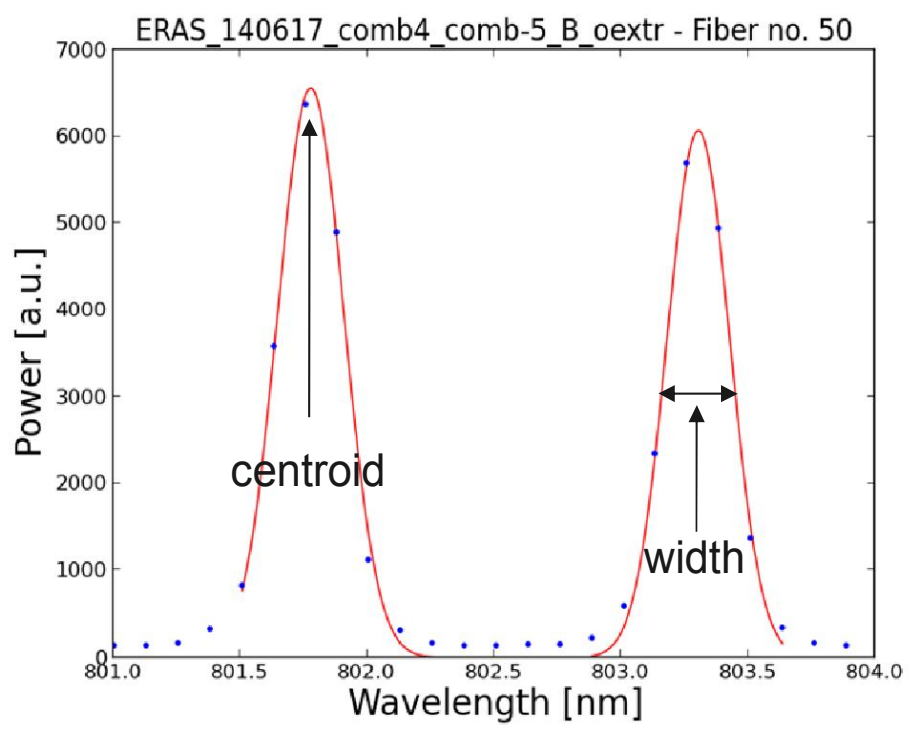

Figure 6. Blue dots: Pixel intensity of two comb lines (centered at $\sim 800 \mathrm{~nm}$ ). Red line: Gaussian fit.

The characteristic that defines an optical frequency comb is that the frequency distance between comb lines is a constant value. If the frequency of one comb line is known and the distance between comb lines is known too, then the frequencies of all the comb lines can be determined. We check the level of accuracy that this equidistancy can be measured using the fiber-fed MUSE spectrograph. The centroid (or central wavelength) is measured for 25 lines and the result is fitted in order to determine the average distance between comb lines and the standard deviation of this distance. Measurements were performed several times in order to check the repeatability of the results. The calculations were done for several fibers chosen randomly from the 400 fibers. The black dots in Figure 7 shows examples of the obtained results for fiber number 50 (left) and 150 (right). The red line illustrates the linear fit performed on the black dots. The average distance between comb lines is $708.5 \mathrm{GHz}$ and the standard deviation is $0.1 \mathrm{GHz}(0.4 \mathrm{pm})$. This value of the standard deviation was found for the other fibers too.
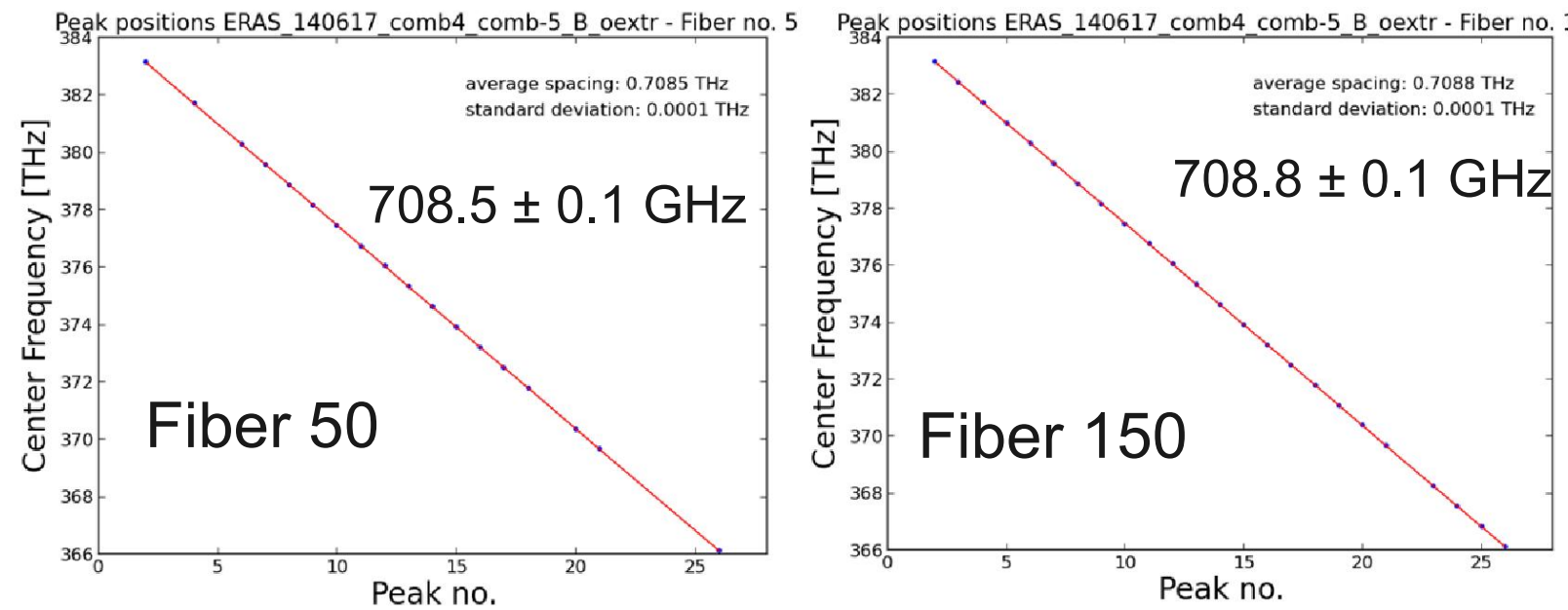

Figure 7. Black dots: center frequency for 25 adjacent comb lines for fiber 50 (left) and 150 (right). Red line: linear fit. 
Table 1 shows a summary of comb spacing and standard deviation measurements of different fibers. The comb spacing is always between 708.5 to $708.8 \mathrm{GHz}$ and the standard deviation $0.1 \mathrm{GHz}(0.4 \mathrm{pm})$. This corresponds to a relative accuracy of $0.14 \%$.

$\begin{array}{lll}\text { Fiber } & \text { Comb spacing } & \text { Standard dev. } \\ 25 & 708.6 \mathrm{GHz} & 0.1 \mathrm{GHz} \\ 45 & 708.7 \mathrm{GHz} & 0.1 \mathrm{GHz} \\ 50 & 708.5 \mathrm{GHz} & 0.1 \mathrm{GHz} \\ 51 & 708.5 \mathrm{GHz} & 0.1 \mathrm{GHz} \\ 55 & 708.5 \mathrm{GHz} & 0.1 \mathrm{GHz} \\ 100 & 708.7 \mathrm{GHz} & 0.1 \mathrm{GHz} \\ 150 & 708.8 \mathrm{GHz} & 0.1 \mathrm{GHz}\end{array}$

This procedure was repeated several times in order to check the stability of the comb and the repeatability of the measurement procedure. The average comb distance and the standard deviation were identical (within $0.3 \mathrm{GHz}$ variation) for different set of measurements.

Figure 8 shows the measured width at full-wave-at-half-maximum for a center wavelength at $802 \mathrm{~nm}$ for the 400 fibers. Note that the fibers at the edge (fibers around 1 or around 400) of $20 \times 20$ arrangement exhibit a larger width when compared the fibers at the center (fiber 200).

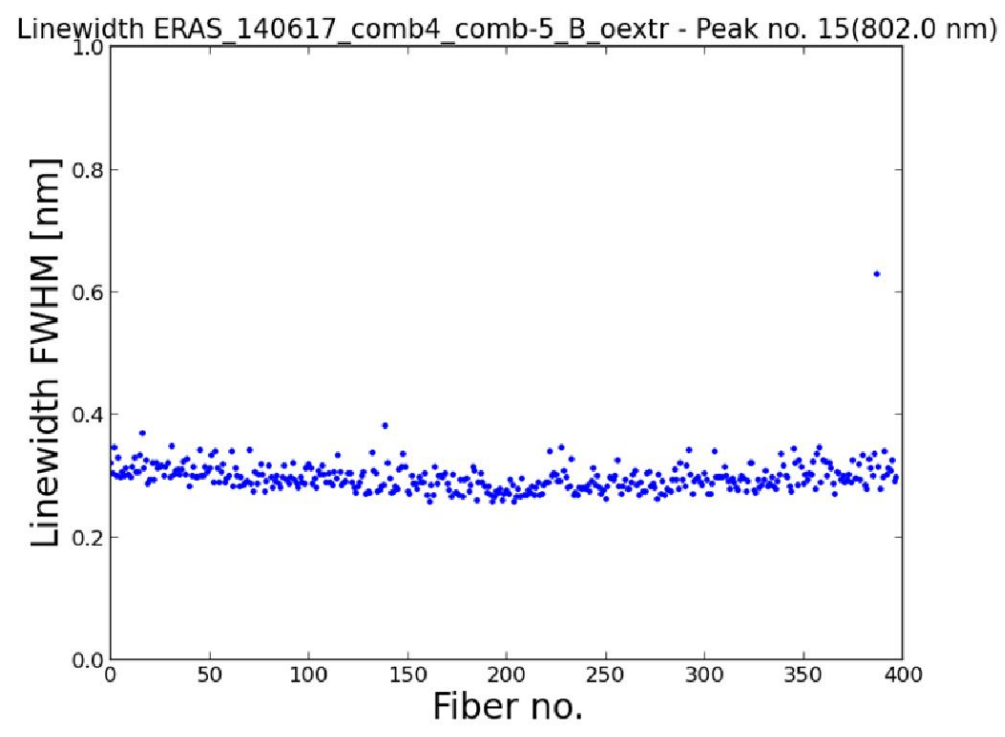

Figure 8. Measured width for a comb line at $802 \mathrm{~nm}$ for the 400 fibers. 


\section{CONCLUSIONS}

In this paper we have described the ongoing research work at innoFSPEC-Potsdam on calibration technologies using astronomical frequency comb generation. The astro-comb is generated by propagating two narrow-linewidth lasers through two dispersion-optimized nonlinear stages. The comb is centered at $1.58 \mu \mathrm{m}$ and comprises 100 calibration lines with good power equalization. The generated comb is transmitted through a BiBO nonlinear crystal where a frequency double process takes place. The frequency doubled comb is centered at $800 \mathrm{~nm}$ and is used to test the MUSE spectrograph. Equidistancy of the comb lines was confirmed within $0.4 \mathrm{pm}$ absolute accuracy.

\section{Acknowledgment}

We would like to acknowledge the financial support from the Federal Ministry of Education and Research (BMBF) for financial support under grants 03Z2AN11 and 03Z2AN12.

\section{REFERENCES}

[1] M.T. Murphy et al., "High-precision wavelength calibration of astronomical spectrographs with laser frequency combs," Mon. Not. R. Astron. Soc., vol. 380, 839-847 (2008).

[2] D. A. Braje, M. S. Kirchner, S. Osterman, T. Fortier and S. A. Diddams, "Astronomical spectrograph calibration with broadspectrum frequency combs", Eur. Phys. J. D, vol. 48, 57-66 (2008).

[3] C. H. Li, A. J. Benedick, P. Fendel, A. G. Glenday, F. X. Kärtner, D. F. Phillips, D. Sasselov, A. Szentgyorgyi and R. L. Walsworth, "A laser frequency comb that enables radial velocity measurements with a precision of $1 \mathrm{~cm} \mathrm{~s}-1$ ", Nature, vol. 452 , 610-612 (2008).

[4] S. L. Redman, G. G. Ycas, R. Terrien, S. Mahadevan, L. W. Ramsey, C. F. Bender, S. N. Osterman, S. A. Diddams, F. Quinlan, J. E. Lawler, and G. Nave, “A high-resolution atlas of uranium-neon in the h band," The Astrophys. J. Suppl. Ser. 199 (2), 1-11 (2012).

[5] K. Griest et al., "Wavelength Accuracy of the Keck HIRES Spectrograph and Measuring Changes in the Fine Structure Constant," Astrophys. J., vol. 708, 158-170 (2010).

[6] A. Loeb, "Direct Measurement of Cosmological Parameters from the Cosmic Deceleration of Extragalactic Objects," Astrophys. J., vol. 499, L111 (1998).

[7] T. Wilken, C. Lovis, A. Manescu, T. Steinmetz, L. Pasquini, et al., "High precision calibration of spectrographs," Mont. Not. R. Astron. Soc., 405, L16-L20 (2010).

[8]T. Steinmetz, et al. "Laser frequency combs for astronomical observations," Science, vol. 321, 1335-1337 (2008).

[9]T. Steinmetz, T. Wilken, C. Araujo-Hauck, R. Holzwarth, T.W. Hänsch and T. Udem, "Fabry-Pérot filter cavities for widespaced frequency combs with large spectral bandwidth", Appl. Phys. B, vol. 96, 251-256 (2009).

[10] T. Wilken, T.W. Hänsch and T. Udem, T. Steinmetz, R. Holzwarth, A. Manescu, G. LoCurto, L. Pasquini, C. Lovis, "High precision calibration of spectrographs in astronomy," Conference on Lasers and Electro-Optics (CLEO), San Jose, CA, USA (2010) .

[11] A. Manescu, et al., "Approaching cm/s calibration of high resolution astronomical spectrographs," CLEO-Europe, Munich (2011).

[12] D.F. Philips et al., "Calibration of an astrophysical spectrograph below $1 \mathrm{~m} / \mathrm{s}$ using a laser frequency comb," Optics Express, Vol. 20, Issue 13, pp. 13711-13726 (2012).

[13] F. Wildi, F. Pepe, B. Chazelas, G. Lo Curto, Ch. Lovis, "A Fabry-Perot calibrator of the HARPS radial velocity spectrograph: performance report, Ground-based and Airborne Instrumentation for Astronomy III. Edited by McLean, Ian S.; Ramsay, Suzanne K.; Takami, Hideki. Proceedings of the SPIE, Volume 7735, pp. 77354X-77354X-11 (2010). [14] P. Del'Haye, A. Schliesser, O. Arcizet, T. Wilken, R. Holzwarth, and T.J. Kippenberg, "Optical frequency comb generation from a monolithic micro-resonator," Nature, vol. 450, 1214-1217 (2007).

[15] S. A. Cerqueira, J. D. Marconi, A. A. Rieznik, H. E. Hernandez-Figueroa, H. L. Fragnito, and J. C. Knight. "Multiple fourwave mixing in ultra-flattened dispersion photonic crystal fibers”. In: OFC/NFOEC. 2008

[16] F. C. Cruz. "Optical frequency combs generated by four-wave mixing in optical fibers for astrophysical spectrometer calibration and metrology”. In: Optics Express 16 (2008), pp. 13267-13275. 